\title{
A. De Saulce, V. Serna. et A. Gallicé (dir.), Archéologies en Loire. Actualité de la recherche dans les régions Centre et Pays-de-la-Loire, Cordemais, Aestuaria-stuarium*
}

Charles-Tanguy Le Roux

\section{(2) OpenEdition}

\section{Journals}

Édition électronique

URL : http://journals.openedition.org/rao/500

DOI : $10.4000 /$ rao. 500

ISBN : 978-2-7535-1607-6

ISSN : 1775-3732

Éditeur

Presses universitaires de Rennes

\section{Édition imprimée}

Date de publication : 30 décembre 2007

Pagination : 218-219

ISBN : 978-2-7535-0574-2

ISSN : 0767-709X

Référence électronique

Charles-Tanguy Le Roux, « A. De Saulce, V. Serna. et A. Gallicé (dir.), Archéologies en Loire. Actualité de la recherche dans les régions Centre et Pays-de-la-Loire, Cordemais, Aestuaria-stuarium* », Revue archéologique de l'Ouest [En ligne], 24 | 2007, mis en ligne le 30 décembre 2009, consulté le 04 décembre 2020. URL : http://journals.openedition.org/rao/500 ; DOI : https://doi.org/10.4000/rao.500 
être entrevues à l'occasion du passage de l'art de bâtir roman (hérité sur place depuis la tradition antique) à la technologie gothique (importée), avec tous ses développements : rôle du fer (chaînages, goujons et agrafes), transposition à la pierre des techniques d'assemblage issues de la charpenterie (application des techniques de charpenterie à la pierre, art du trait et stéréotomie, évacuation des eaux, etc.). Pour finir, sont évoqués les questionnements relatifs aux restaurations et transformations; ne l'oublions pas, " un édifice est un bâti en constante évolution » dont " la restauration a commencé dès le Moyen-Âge » (p. 316).

L'ouvrage est complété par deux « annexes » : 1) les arcs intégrés dans l'espace rhodano-méditerranéen (un inventaire qui renvoie à la carte de la fig. 152 dans le corps de l'ouvrage) et 2) une étude des échafaudements pour la pose des remplages et le levage des vitraux de Saint-Nizier (par C. Le Barrier). Il se termine comme il se doit par une copieuse bibliographie qui distingue les "sources archéologiques " (c'est-à-dire les rapports d'opérations), les « sources écrites » et la «bibliographie » archéologique proprement dite et se prolonge, in fine, par des résumés (en français, italien et anglais).

C'est là un livre dense et copieux mais fort bien présenté, abondamment illustré (dessins, plans, axonométries et photographies, pour partie en couleurs, sont de belle venue) et agréable à lire. Une mine de renseignements pour les archéologues médiévistes et les architectes restaurateurs, un prodigieux terrain de découverte pour les autres.

La rédaction

de Saulce, A., Serna, V. et Galuicé, A. (dir.), 2007 - Archéologies en Loire. Actualité de la recherche dans les régions Centre et Pays-de-la-Loire, Cordemais, Aestuaria-Estuarium*, 416 p. (ISBN 978-2-9528512-4-4; $30 €$ ).

Ce deuxième volume de la collection «Fleuves et Archéologie " s'attache à présenter une série d'opérations très variées concernant la vallée de la Loire ou la basse vallée de ses affluents; il a été coordonné par les Services régionaux de l'Archéologie des deux régions concernées. Comme le soulignent L. Bourgeau et B. Mandy dans leur préface, la Loire, qui est pourtant un élément structurant majeur d'une bonne partie de l'actuel territoire français, "a longtemps souffert de l'absence d'études transversales "; à côté des quelques travaux précurseurs évoqués à cette occasion, il serait cependant injuste d'oublier l'ouvrage de M. Provost (1993). Mais le récent classement au Patrimoine mondial par l'UNESCO " a permis de nouvelles interventions dans le cadre du volet culturel du Plan Loire " et les 21 contributions rassemblées ici constituent un premier bilan impressionnant des potentialités de cette zone. La plupart correspondent à des opérations menées en 2003-2006, mais la dynamique ainsi créée a permis de réveiller quelques "belles au bois dormant" oubliées, comme l'habitat Bronze final de l'Alleu, aux portes de Saumur, resté quasi-inédit depuis 1977.

Le cœur de l'ouvrage commence par la présentation de deux PCR en cours: "Navigation et navigabilité sur les petites rivières en région Centre " (V. Serna) et « Des Ponts-de-Cé à l'estuaire : interactions Homme/Milieu de la Loire et de ses

\footnotetext{
* Diffusion : Littéral, ZI du Bois-Lambert, 85280 La Ferrière.

Provost, M., 1993 - Le Val de Loire dans l'Antiquité, Paris, CNRS, (52 suppl. à Gallia), 426 p.

Le Roux, C.-T., 1999 - Loutillage de pierre polie en métadolérite du type A, Rennes, université de Rennes 1 (Travaux du laboratoire "Anthropologie, Préhistoire et Quaternaire armoricains ", 43), 244 p.
}

affluents" (A. de Saulce), puis il s'organise autour de quatre grands thèmes : «Espace navigué, fleuve aménagé ", «Traverser la Loire ", "Archéologie préventive dans le bassin de la Loire " et "Enquêtes, chantiers et découvertes fortuites".

Dans chacune de ces rubriques, on trouve deux types d'études. Quelques sujets sont abordés de manière « extensive » : La batellerie monoxyle (G. Creïs et al.), Cales et quais (O. Cléricy et al.), Les franchissements (E. Miejac et A. de Saulce), Les alluvions fossiles (R. Arthuis et al.), Les dépôts à l'âge du Bronze (M. Melin). Cependant, la plupart des études restent fondamentalement monographiques, montrant bien que le temps des brillantes synthèses n'est pas encore venu; la qualité et la variété de ces travaux et les horizons qu'ils nous ouvrent laissent cependant augurer du meilleur pour un proche avenir.

Il n'est pas possible ici de résumer toutes les contributions et, à l'inverse, il serait injuste de n'en mettre que certaines en exergue; notons cependant, au fil des pages, quelques-uns des faits marquants qui y sont présentés.

- L'interrogation de G. Creïs et al. sur l'étonnante rareté des épaves anciennes signalées au fil des $400 \mathrm{~km}$ étudiés d'un fleuve où la navigation a toujours été intense.

- La réinterprétation (terre-plein précédé d'un radier plutôt que quai) des aménagements de berge au droit des entrepôts antiques de Rezé (R. Arthuis et al.).

- Les enjeux politiques et militaires liés aux franchissements de la Loire (E. Miejac et A. de Saulce) - comme quoi l'on voit que l'épisode des "Cadets de Saumur", en 1940, s'inscrit dans une bien longue histoire (au fait, que reste-t-il comme traces étudiables de ces récents combats sur le terrain?). 
- La précocité des premiers ponts sur le fleuve - dès le tout début du i ${ }^{\mathrm{er}}$ siècle de notre ère (A. Dumont et al.).

- La difficile confrontation (une de plus...) entre la tradition, les sources écrites et les faits archéologiques à propos des installations Vikings face à l'abbaye de Saint-Florent (M. Rolland).

- L'importance de l'habitat Bronze final de l'Alleu à SaintHilaire-Saint-Florent, le plus important de faciès "RhinSuisse-France orientale " dans toute la moitié ouest de la France (T. Nicolas). C'est là un témoignage de plus, s'il en était besoin, pour affirmer l'importance de l'axe ligérien dès la protohistoire (et même sans doute bien avant : entre autres, l'auteur de ces lignes a déjà eu l'occasion de "pister " les lames de haches polies néolithiques le long du Val de Loire, aussi bien depuis le Massif armoricain que depuis les régions alpines : Le Roux 1999 - cf. notamment les fig. 59-60 et 69).

- N'oublions pas enfin l'histoire sédimentaire et morphologique complexe du fleuve, de son lit et de ses îles, de ses ensablements et variations de niveau depuis la préhistoire jusqu'à nos jours, qui transparaît dans plusieurs contributions.

En résumé, nous avons là un magistral « état de la question " sur une problématique pleine de promesses; la matière certes aspire à être complétée, mais les données présentées ici sont indiscutablement destinées à lancer une dynamique de recherche; elles sont aussi appelées à faire référence pendant " un certain temps » encore.

Charles-Tanguy LE Roux

SAujot, C., 2007 - Le droit français de l'archéologie, Paris, Cujas, $2^{e}$ édition, 352 p. (ISBN 978-2-254-08003-8; $45 €$ )

Ce livre est la seconde édition d'un ouvrage dont la première édition, datée de 2003 mais parue au début de l'année 2004, avait en son temps donné lieu à compte rendu dans ces colonnes (RAO, t. 20, 2003, p. 247-248). Depuis 2003, le paysage législatif français a beaucoup évolué, et par voie de conséquence, la jurisprudence y afférant. Aussi, pour cette seconde édition, l'auteur a-t-elle entièrement refondue et mise à jour la première version : les derniers textes législatifs et la jurisprudence pris en compte vont jusqu'au $1^{\text {er }}$ septembre 2007. Il serait difficile de présenter davantage plus à jour, pour un ouvrage sorti des presses en octobre 2007!

L'auteur, éminente juriste et docteur en archéologie, est bien connue des lecteurs pour ses pertinentes chroniques du droit de l'archéologie régulièrement publiées dans la $R A O$.

La première partie de l'ouvrage présente la recherche des vestiges, à savoir la législation des fouilles terrestres et subaquatiques et la question des découvertes fortuites, donc la définition juridique du "trésor ". L'archéologie préventive occupe, ensuite, une place importante dans l'ouvrage. S'il est un domaine dans lequel les choses ont beaucoup évolué, c'est bien celui-ci!

La seconde partie de l'ouvrage est consacrée au statut des vestiges, meubles comme immeubles. C'est là une partie particulièrement importante de l'ouvrage, à une époque où les conditions de propriété des vestiges ont considérablement évolué (chacun a en mémoire l'affaire de la grotte Chauvet), et où les collections publiques des musées nationaux et des collectivités territoriales, comme celles d'associations bénéficiant du statut de Musée de France, s'attachent à régler de façon claire et définitive le statut légal des biens qu'ils détiennent, ou sont appelés à recevoir.
La troisième partie traite de la protection des vestiges. La question des fouilles illégales est traitée ici. Des pages que feraient bien de méditer les utilisateurs clandestins de détecteurs à métaux... Les monuments historiques classés ou inscrits à l'Inventaire supplémentaire des $\mathrm{MH}$, bâtis comme objets mobiliers, font l'objet du dernier chapitre.

En sus d'une considérable documentation donnée en notes infrapaginales, une importante bibliographie complète l'ouvrage.

Cette nouvelle version de l'ouvrage de $\mathrm{M}^{\text {me }}$ Saujot rendra les plus distingués services à tous ceux que concerne la recherche archéologique et la défense de notre patrimoine, qui seront désormais bien armés : personnels des Services régionaux de l'Archéologie, professions juridiques, chercheurs institutionnels, mais aussi bénévoles, en particulier les membres des " associations loi de 1901 ». On l'oublie trop, ces dernières sont susceptibles, lorsque leurs statuts prévoient la défense du patrimoine historique et/ou archéologique, d'ester devant la justice civile. L'efficacité de cette démarche n'est plus à démontrer!

José Gomez de Soto

(Directeur de recherche, UMR 6566) 